\title{
One Electron Molecules with Relativistic Kinetic Energy: Properties of the Discrete Spectrum
}

\section{Ingrid Daubechies*}

Theoretische Natuurkunde, Vrije Universiteit Brussel, Pleinlaan 2, B-1050 Brussel, Belgium

Abstract. We discuss the discrete spectrum of the operator

$$
H_{K}(c)=\left[-\hbar^{2} c^{2} \Delta+m^{2} c^{4}\right]^{1 / 2}-\sum_{k=1}^{K} Z_{k} e^{2}\left|x-R_{k}\right|^{-1} .
$$

More specifically, we study 1) the behaviour of the eigenvalues when the internuclear distances contract, 2) the existence of a $c$-independent lower bound for $H_{K}(c)-m c^{2}$, 3) the nonrelativistic limit of the eigenvalues of $H_{K}(c)-m c^{2}$.

\section{Introduction}

This paper deals with the operator

$$
H_{K}=\left(-\hbar^{2} c^{2} \Delta+m^{2} c^{4}\right)^{1 / 2}-\sum_{k=1}^{K} Z_{k} e^{2}\left|x-R_{k}\right|^{-1}
$$

describing a relativistic charged particle with mass $m$ in the presence of $K$ fixed nuclei (Born-Oppenheimer approximation). The kinetic energy operator for the charged particle is obtained by straightforward "quantization" of the relativistic formula for the kinetic energy $\left[p^{2} c^{2}+m^{2} c^{4}\right]^{1 / 2} ;(1)$ can be considered as an alternative to the Klein-Gordon equation for a relativistic model neglecting spin effects. In what follows we shall, with a slight abuse of terminology, use the name "electron" for the charged particle described by (1).

For the case $K=1$ the above operator has been studied in detail by Herbst [1] and Weder [2]. One finds that

$$
H_{1}=\left(-\hbar^{2} c^{2} \Delta+m^{2} c^{4}\right)^{1 / 2}-Z e^{2}|x|^{-1}
$$

is bounded below if and only if the nuclear charge $Z$ is less than a critical value $Z_{\text {crit }}$. This phenomenon is typical for relativistic atom models; the value for $Z_{\text {crit }}$ in the

* "Wetenschappelijk medewerker" at the Interuniversitair Instituut voor Kernwetenschappen, Belgium 
present case, $Z_{\text {crit }}=2 \alpha^{-1} / \pi \quad$ [where $\left.\alpha=e^{2} / \hbar c \approx(137)^{-1}\right]$, lies between the corresponding critical values for the Dirac and Klein-Gordon theories (where $Z_{\text {crit }}=\alpha^{-1}$ and $\alpha^{-1} / 2$, respectively). For $Z<Z_{\text {crit }}$ the discrete spectrum of $H_{1}$ consists of infinitely many eigenvalues between 0 and $m c^{2}$, accumulating at $m c^{2}$; the remainder of the spectrum is purely absolutely continuous and consists of the half line $\left[m c^{2}, \infty\right)$. Moreover [1], the eigenvalues of $H_{1}$ are separated from 0 by a gap increasing with $Z-Z_{\text {crit }}$ :

$$
\sigma_{\text {disc }}\left(H_{1}\right) \subset\left[\left(1-Z^{2} / Z_{\text {crit }}^{2}\right)^{1 / 2} m c^{2}, m c^{2}\right) .
$$

In the nonrelativistic limit, this gives a lower bound on the difference between the ground state energy $E_{0}$ of $H_{1}$ and the rest energy $m c^{2}$ :

$$
E_{0}\left(H_{1}\right)-m c^{2} \geqq-\left(\pi^{2} / 8\right) Z^{2} e^{4} m \hbar^{-2}+O\left(c^{-2}\right) .
$$

Up to terms of order $c^{-2}$ this lower bound is independent of $c$; it has the same dependence on $Z, e, m$, and $\hbar$ as the ground state of the true hydrogenic atom [the coefficient $\pi^{2} / 8$ is larger than the true coefficient $1 / 2$, but after all, (3) only gives a lower bound].

For arbitrary values of $K$, a first study of $H_{K}$ was made in [3]. As in the case $K=1$, the operator $H_{K}$ is bounded below if and only if $Z_{k} \leqq Z_{\text {crit }}=2 \alpha^{-1} / \pi$, for all $k$. We shall always restrict ourselves to this case. Again one finds for $Z_{k}<Z_{\text {crit }}$, that the essential spectrum of $H_{K}$ is the half-line $\left[m c^{2}, \infty\right)$. If $\sum_{k=1}^{K} Z_{k}>Z_{\text {crit }}$, negative eigenvalues occur [unlike the $K=1$ case: see (2)], which may be made arbitrarily negative by bringing the nuclei close enough together. This "collapsing" tendency is, however, held in check by the electrostatic repulsion between the nuclei: it was proved in [3] that the total energy (including the electrostatic repulsion between the nuclei) for the ground state of the one electron $-K$ nucleus - system is positive:

$$
E_{0}\left(H_{K}\right)+\sum_{\substack{k, l=1 \\ k<l}}^{K} Z_{k} Z_{l} e^{2}\left|R_{k}-R_{l}\right|^{-1} \geqq 0 .
$$

This means that the system is stable (see [3]).

In the present paper we want to address three further questions concerning the spectrum of $\left.H_{K}: 1\right)$ the behaviour of the energy levels when the internuclear distances $\left|R_{k}-R_{l}\right|$ tend to zero, 2) the existence of a lower bound analoguous to (2), for the case $K \neq 1$, and 3) the non-relativistic limit of the eigenvalues of $H_{K}$.

When the electron mass is put equal to zero, $m=0$, it is easy to see what happens if the $\left|R_{k}-R_{l}\right|$ all shrink to zero. If $\sum_{k=1}^{K} Z_{k}>Z_{\text {crit }}$, one finds that $h_{K}(\mathbf{R})$ $=H_{K}(m=0 ; \mathbf{R})$ has non-empty discrete spectrum (we use the notation $\mathbf{R}$ for the set $\left.\left\{R_{k}\right\}_{k=1, \ldots, K}\right)$. Since $h_{K}(\mathbf{R})$ and $\lambda h_{K}(\lambda \mathbf{R})$ are unitarily equivalent, the eigenvalues $e_{n}(\mathbf{R})$ of $h_{K}$ have the property

$$
e_{n}(\lambda \mathbf{R})=\lambda^{-1} e_{n}(\mathbf{R}) .
$$

As $\lambda$ tends to zero, the eigenvalues of $h_{K}(\lambda \mathbf{R})$ all tend to $-\infty$. We prove in Sect. 2 that a similar phenomenon takes place if the electron mass is different from zero: 
Theorem 1. Let $H_{K}$ be defined as in (1), with $0 \leqq Z_{k} \leqq Z_{\text {crit }}$ for all $k$. Suppose that $\sum_{k=1}^{K} Z_{k}>Z_{\text {crit. }}$ Then $\forall E<0, \forall N_{0} \in \mathbb{N}: \exists \lambda_{0}$ such that

$$
\lambda \leqq \lambda_{0} \Rightarrow \#\left\{E_{n} ; E_{n} \text { eigenvalue of } H_{K}(\lambda \mathbf{R}), E_{n}<E\right\} \geqq N_{0} .
$$

A similar theorem for the Dirac operator with several Coulomb singularities, where $E$ can be chosen arbitrarily in ] $-m c^{2}, m c^{2}$, was proved by Klaus [4]. Our proof of the above theorem is inspired by the proofs given in [4]: the essential ingredients are the use of the Birman-Schwinger-kernel, and an argument using strong resolvent convergence.

In Sect. 3 we prove a lower bound for $H_{K}$ analogous to (2). The following simple argument already gives such a lower bound. For fixed $Z_{1}, \ldots, Z_{K}$, there exists a value $c_{0}$ of $c$ such that $\sum_{k=1}^{K} Z_{k} \leqq(2 / \pi) \hbar c_{0} / e^{2}$. The same argument used by Herbst in [1] to obtain (2) can then be applied in the present case, and we have $\left(Z_{\text {tot }}=\sum_{k} Z_{k}\right)$ :

$$
c \geqq c_{0} \Rightarrow \sigma_{\text {disc }}\left(H_{K}\right) \subset\left[\left(1-Z_{\text {tot }}^{2} / Z_{\text {crit }}^{2}\right)^{1 / 2} m c^{2}, m c^{2}\right)
$$

[where again $Z_{\text {crit }}=(2 / \pi)\left(e^{2} / \hbar c\right)^{-1}$ ]. This lower bound seems to be non-optimal from two points of view:

1) It is only valid for large enough values of $c$; the lower limit $c_{0}$ depends on the choice for the $Z_{k}$. It would be rather surprising if this were the best one can do.

2) If the nuclei are widely enough separated, one would expect the electron to settle around the nucleus with the largest charge without "seeing" the other nuclei. This would be reflected by a dependence of the lower bound on $Z_{\max }=\max \left\{Z_{k} ; k=1, \ldots, K\right\}$ rather than on $Z_{\text {tot }}$. Both these criticisms of (6) are avoided by the following theorem, proved in Sect. 3:

Theorem 2. For any $K$, and any $Z_{1} \ldots Z_{K}$ with

$$
6 \leqq \max _{k} Z_{k} \leqq(2 / \pi) \hbar c / e^{2}=Z_{\text {crit }}
$$

we have

$$
H_{K}+\sum_{\substack{k, l=1 \\ k<l}}^{K} Z_{k} Z_{l} e^{2}\left|R_{k}-R_{l}\right|^{-1} \geqq m c^{2}\left[1-\left(\max Z_{k}\right)^{2} / Z_{\mathrm{crit}}^{2}\right]^{1 / 2} .
$$

For $\max Z_{k}<6$ we prove a weaker result (see Sect. 3). The proof of(7) uses a lower bound similar to but stronger than (4), which was also proved in [3]. Note that (7) contains the electrostatic repulsion between the nuclei: if the nuclei are far apart, this term becomes negligible, and (7) is a much better bound than (6); if the nuclei are very close however, the repulsion energy becomes rather large, and (6) may be better than (7).

Finally, in Sect. 4, we study the nonrelativistic limit (i.e. $c \rightarrow \infty$ ) of the eigenvalues of $H_{K}(c)$. The lower bounds (6) and (7) already imply a nonrelativistic lower bound for the ground state energy $E_{0}\left(H_{K}\right)-m c^{2}$, similar to (3). As we noted 
above, these nonrelativistic lower bounds for the ground state energy are too small by a factor $\pi^{2} / 4$ when compared with the expected limit. Moreover, these lower bounds cannot give us any information concerning the excited states. We prove in Sect. 4 that all the energy levels of $H_{K}$ tend to the corresponding bound state energy levels of the nonrelativistic Hamiltonian when $c \rightarrow \infty$ :

Theorem 3. Define

$$
H_{K}^{0}=-\left(\hbar^{2} / 2 m\right) \Delta-\sum_{k=1}^{K} Z_{k} e^{2}\left|x-R_{k}\right|^{-1} .
$$

Let $E_{n}(c), E_{n}^{0}$ respectively be the $n^{\text {th }}$ eigenvalues (counting multiplicity) of $H_{K}(c), H_{K}^{0}$, where $H_{K}(c)$ is given by $(1)$. Then $\lim _{c \rightarrow \infty}\left[E_{n}(c)-m c^{2}\right]=E_{n}^{0}$.

To prove this, we show that there exists a $c$-independent $z$ in $\mathbb{C}$ such that the resolvent $\left(H_{K}(c)-m c^{2}-z\right)^{-1}$ is norm continuous in $c^{-1}$ around the nonrelativistic limit $c^{-1}=0$. The proof uses the fact that (7) implies the existence of a $c$-independent lower bound for $H_{K}(c)-m c^{2}$.

\section{The Behaviour of the Eigenvalues under Contractions}

For this and the next section we shall use units such that $\hbar=c=1$. We can then rewrite $H_{K}$ as

$$
H_{K}=\left(p^{2}+m^{2}\right)^{1 / 2}-V_{\mathbf{R}}(x)
$$

where $p^{2}=-\Delta$, and

$$
V_{\mathbf{R}}(x)=\sum_{k=1}^{K} Z_{k}\left(e^{2} / \hbar c\right)\left|x-R_{k}\right|^{-1}=(2 / \pi) \sum_{k=1}^{K} \mu_{k}\left|x-R_{k}\right|^{-1}
$$

with $\mu_{k}=Z_{k} / Z_{\text {crit }}$.

We consider the situation in which $M \equiv \sum_{k=1}^{K} \mu_{k}>1$; as always we have $\mu_{k} \leqq 1$ for all $k$, hence $\mu \equiv \max _{k} \mu_{k} \leqq 1$.

According to the Birman-Schwinger principle, we have, for $E<0$,

$$
\begin{aligned}
\# & \left\{E_{n} ; E_{n} \text { eigenvalue of } H_{K}, E_{n}<E\right\} \\
& =\#\left\{e_{n} ; e_{n} \text { eigenvalue of } V_{\mathbf{R}}^{1 / 2}\left[\left(p^{2}+m^{2}\right)^{1 / 2}+|E|\right]^{-1} V_{\mathbf{R}}^{1 / 2}, e_{n}>1\right\} .
\end{aligned}
$$

We shall therefore make a study of the spectrum of

$$
V_{\mathbf{R}}^{1 / 2}\left[\left(p^{2}+m^{2}\right)^{1 / 2}+|E|\right]^{-1} V_{\mathbf{R}}^{1 / 2} .
$$

We shall show that on the one hand

$$
\sigma_{\text {ess }}\left(V_{\mathbf{R}}^{1 / 2}\left[\left(p^{2}+m^{2}\right)^{1 / 2}+|E|\right]^{-1} V_{\mathbf{R}}^{1 / 2}\right)=[0, \mu],
$$

while on the other hand every point in $[0, M]$ is an accumulation point of

$$
\bigcup_{n} \sigma\left(V_{\mathbf{R} / n}^{1 / 2}\left[\left(p^{2}+m^{2}\right)^{1 / 2}+|E|\right]^{-1} V_{\mathbf{R} / n}^{1 / 2}\right)
$$


(see below). Since $M>1 \geqq \mu$, this will imply that the number of eigenvalues greater than 1 of the operator $V_{\mathbf{R} / n}^{1 / 2}\left[\left(p^{2}+m^{2}\right)^{1 / 2}+|E|\right]^{-1} V_{\mathbf{R} / n}^{1 / 2}$ tends to infinity for $n \rightarrow \infty$.

We first compute the spectrum of $|x|^{-1 / 2}|p|^{-1}|x|^{-1 / 2}$, where $|p|=(-\Delta)^{1 / 2}$.

Lemma. $\sigma\left(|x|^{-1 / 2}|p|^{-1}|x|^{-1 / 2}\right)=\sigma_{\mathrm{ac}}\left(|x|^{-1 / 2}|p|^{-1}|x|^{-1 / 2}\right)=[0, \pi / 2]$.

Proof. Since $|x|^{-1 / 2}|p|^{-1}|x|^{-1 / 2} \geqq 0$, and $\left\||p|^{-1 / 2}|x|^{-1 / 2}\right\|=(\pi / 2)^{1 / 2}$ (see [1]), we obviously have $\sigma\left(|x|^{-1 / 2}|p|^{-1}|x|^{-1 / 2}\right) \subset[0, \pi / 2]$. We prove that this inclusion is an equality by explicit computation. On $L^{2}\left(\mathbb{R}^{3}\right)$, the operator $|x|^{-1 / 2}|p|^{-1}|x|^{-1 / 2}$ has integral kernel

$$
\left(2 \pi^{2}\right)^{-1}|x|^{-1 / 2}|x-y|^{-2}|y|^{-1 / 2} \text {. }
$$

Defining the unitary operator $U$ from $L^{2}\left(\mathbb{R}^{3}\right)$ to $L^{2}\left(\mathbb{R} \times S^{2}\right)$ by

$$
(U f)(t, \omega)=e^{3 t / 2} f\left(e^{t} \omega\right),
$$

one finds that on $L^{2}\left(\mathbb{R} \times S^{2}\right)$ the operator

$$
U|x|^{-1 / 2}|p|^{-1}|x|^{-1 / 2} U^{-1}
$$

is given by the integral kernel

$$
F\left(t_{1}, t_{2} ; \omega_{1}, \omega_{2}\right)=\left(4 \pi^{2}\right)^{-1}\left[\cosh \left(t_{1}-t_{2}\right)-\omega_{1} \cdot \omega_{2}\right]^{-1} .
$$

Since $F$ depends only on the difference $t_{1}-t_{2}$, we see that by a Fourier transform $|x|^{-1 / 2}|p|^{-1}|x|^{-1 / 2}$ is unitarily equivalent to the operator $B$ on $L^{2}\left(\mathbb{R} \times S^{2}\right)$ defined by

$$
(B f)(k, \omega)=\int d \omega^{\prime} b\left(k ; \omega, \omega^{\prime}\right) f\left(k, \omega^{\prime}\right)
$$

with

$$
\begin{aligned}
b\left(k ; \omega, \omega^{\prime}\right) & =\int d t e^{i k t} F\left(t, 0 ; \omega, \omega^{\prime}\right) \\
& =(2 \pi)^{-1}\left[1-\left(\omega \cdot \omega^{\prime}\right)^{2}\right]^{-1 / 2} \frac{\sinh \left[k\left(\pi-\cos ^{-1} \omega \cdot \omega^{\prime}\right)\right]}{\sinh k \pi},
\end{aligned}
$$

where we choose $\cos ^{-1} \omega \cdot \omega^{\prime} \in[0, \pi]$.

Since the integral kernel $b\left(k ; \omega, \omega^{\prime}\right)$ depends only on $\omega \cdot \omega^{\prime}, B$ can be written as a direct sum $\bigoplus_{l=0}^{\infty} \bigoplus_{m=-l}^{l} B_{l m}$, where each $B_{l m}$ acts on $L^{2}(\mathbb{R})$ and is given by an integral kernel too:

$$
\left(B_{l m} f\right)(k)=b_{l m}(k) f(k),
$$

with

$$
b_{l m}(k)=\int_{S^{2}} d \omega_{1} \int_{S^{2}} d \omega_{2} \overline{Y_{l m}\left(\omega_{1}\right)} Y_{l m}\left(\omega_{2}\right) b\left(k ; \omega_{1}, \omega_{2}\right) .
$$

One can check that each of these functions $b_{l m}$ is continuous, tending to zero for $k \rightarrow \infty$. This implies that $|x|^{-1 / 2}|p|^{-1}|x|^{-1 / 2}$ has only absolutely continuous spectrum. Moreover one finds

$b_{00}(0)=\pi / 2$, hence $b_{00}(\mathbb{R})=[0, \pi / 2]$, which implies

$$
\sigma\left(|x|^{-1 / 2}|p|^{-1}|x|^{-1 / 2}\right) \supset \sigma\left(B_{00}\right)=b_{00}(\mathbb{R})=[0, \pi / 2] .
$$

In the following proposition we prove how this implies (10). 
Proposition. Let $V_{\mathbf{R}}$ be defined as in (9), with $R_{k} \neq R_{l}$ for $k \neq l$. Then

$$
\begin{gathered}
\sigma_{\text {ess }}\left(V_{\mathbf{R}}^{1 / 2}\left[\left(p^{2}+m^{2}\right)^{1 / 2}+|E|\right]^{-1} V_{\mathbf{R}}^{1 / 2}\right)=\sigma_{\text {ess }}\left(V_{\mathbf{R}}^{1 / 2}(|p|+1)^{-1} V_{\mathbf{R}}^{1 / 2}\right) \\
=(2 / \pi) \mu \sigma_{\text {ess }}\left(|x|^{-1 / 2}|p|^{-1}|x|^{-1 / 2}\right)=[0, \mu] .
\end{gathered}
$$

Proof. For $B_{1}, B_{2}$ bounded operators, we shall use the notation $B_{1}={ }_{c} B_{2}$ if $B_{1}-B_{2}$ is compact. Choose a strictly positive $\delta$ such that $2 \delta<\left|R_{k}-R_{l}\right|$ for all $k \neq l$. Define $f_{k}(x)=1$ if $\left|x-R_{k}\right| \leqq \delta, f_{k}(x)=0$ otherwise. Define $f=\sum_{k} f_{k}$. Since $(|p|+1)^{-1 / 2} V_{\mathbf{R}}^{1 / 2}$ is bounded, we have $(n-\lim =$ norm limit $)$

$$
(|p|+1)^{-1} V_{\mathbf{R}}^{1 / 2}=n-\lim _{\varepsilon \rightarrow 0} e^{-\varepsilon p^{2}}(|p|+1)^{-1} V_{\mathbf{R}}^{1 / 2} .
$$

Moreover

$$
e^{-\varepsilon p^{2}}(|p|+1)^{-1} V_{\mathbf{R}}^{1 / 2}=n-\lim _{\lambda \rightarrow 0} e^{-\varepsilon p^{2}}(|p|+1)^{-1}\left[f(x) V_{\mathbf{R}}^{1 / 2}+V_{\mathbf{R}}^{1 / 2}(1-f(x)) e^{-\lambda x^{2}}\right]
$$

is a compact operator (as the norm limit of Hilbert-Schmidt operators), which implies that $(|p|+1)^{-1} V_{\mathbf{R}}^{1 / 2}$ is compact. Since

$$
|E|-1 \leqq\left(p^{2}+m^{2}\right)^{1 / 2}+|E|-(|p|+1) \leqq|E|+m-1,
$$

we have therefore

$$
V_{\mathbf{R}}^{1 / 2}\left[\left(p^{2}+m^{2}\right)^{1 / 2}+|E|\right]^{-1} V_{\mathbf{R}}^{1 / 2}={ }_{c} V_{\mathbf{R}}^{1 / 2}(|p|+1)^{-1} V_{\mathbf{R}}^{1 / 2}
$$

this proves the first equality of the proposition (by Weyl's theorem). Since $(1-f) V_{\mathbf{R}}^{1 / 2}$ is bounded, we have also

$$
V_{\mathbf{R}}^{1 / 2}(|p|+1)^{-1} V_{\mathbf{R}}^{1 / 2}={ }_{c} f V_{\mathbf{R}}^{1 / 2}(|p|+1)^{-1} V_{\mathbf{R}}^{1 / 2} f .
$$

It is easy to check that $f V_{\mathbf{R}}^{1 / 2}-\sum_{k} f_{k}(x)\left(2 \mu_{k} / \pi\right)^{1 / 2}\left|x-R_{k}\right|^{-1 / 2}$ is bounded. Using the fact that $|p|-1$ has integral kernel $\left(2 \pi^{2}\right)^{-1}|x-y|^{-2}$, one sees moreover that

$$
f_{k}\left|x-R_{k}\right|^{-1 / 2}(|p|+1)^{-1}\left|x-R_{l}\right|^{-1 / 2} f_{l}
$$

is Hilbert-Schmidt for $k \neq l$. Hence

$$
f V_{\mathbf{R}}^{1 / 2}(|p|+1)^{-1} V_{\mathbf{R}}^{1 / 2} f={ }_{c}(2 / \pi) \sum_{k} \mu_{k} f_{k}\left|x-R_{k}\right|^{-1 / 2}(|p|+1)^{-1}\left|x-R_{k}\right|^{-1 / 2} f_{k} .
$$

Since the $f_{k}$ are the characteristic functions of disjoint balls, the sum in the right hand member of (12) can be considered as a direct sum of unitarily equivalent operators (up to the coefficients $\mu_{k}$ ). Defining $g(x)=1$ if $|x| \leqq \delta, g(x)=0$ otherwise, we can therefore conclude from (11) and (12) that $\sigma_{\text {ess }}\left(V_{\mathbf{R}}^{1 / 2}(|p|+1)^{-1} V_{\mathbf{R}}^{1 / 2}\right)$ is completely determined by

$$
\sigma\left(g|x|^{-1 / 2}(|p|+1)^{-1}|x|^{-1 / 2} g\right) .
$$

Again, we have that $(1-g)|x|^{-1 / 2}$ is bounded, while $(|p|+1)^{-1}|x|^{-1 / 2}$ is compact, which implies

$$
g|x|^{-1 / 2}(|p|+1)^{-1}|x|^{-1 / 2} g={ }_{c}|x|^{-1 / 2}(|p|+1)^{-1}|x|^{-1 / 2}
$$


Hence

$$
\sigma_{\text {ess }}\left(g|x|^{-1 / 2}(|p|+1)^{-1}|x|^{-1 / 2} g\right)=\sigma_{\text {ess }}\left(|x|^{-1 / 2}(|p|+1)^{-1}|x|^{-1 / 2}\right) .
$$

One easily sees that all the operators $|x|^{-1 / 2}(|p|+\lambda)^{-1}|x|^{-1 / 2}, \lambda>0$, are unitarily equivalent under dilations. This implies that $\sigma\left(|x|^{-1 / 2}(|p|+\lambda)^{-1}|x|^{-1 / 2}\right)$ is independent of $\lambda$. On the other hand $|x|^{-1 / 2}(|p|+\lambda)^{-1}|x|^{-1 / 2}$ converges strongly to $|x|^{-1 / 2}|p|^{-1}|x|^{-1 / 2}$ for $\lambda \rightarrow 0$. This implies that every $e \in \sigma\left(|x|^{-1 / 2}|p|^{-1}|x|^{-1 / 2}\right)$ can be written as $e=\lim _{n \rightarrow \infty} e_{n}$, with

$$
e_{n} \in \sigma\left(|x|^{-1 / 2}(|p|+1 / n)^{-1}|x|^{-1 / 2}\right) .
$$

Combining these two facts gives

$$
[0, \pi / 2]=\sigma\left(|x|^{-1 / 2}|p|^{-1}|x|^{-1 / 2}\right) \subset \sigma\left(|x|^{-1 / 2}(|p|+1)^{-1}|x|^{-1 / 2}\right) .
$$

Since also

$$
0 \leqq|x|^{-1 / 2}(|p|+1)^{-1}|x|^{-1 / 2} \leqq|x|^{-1 / 2}|p|^{-1}|x|^{-1 / 2} \leqq \pi / 2,
$$

we find $\sigma\left(|x|^{-1 / 2}(|p|+1)^{-1}|x|^{-1 / 2}\right)=[0, \pi / 2]$, hence, by (13), and because

$$
\begin{gathered}
0 \leqq g|x|^{-1 / 2}(|p|+1)^{-1 / 2}|x|^{-1 / 2} g \leqq \pi / 2, \\
\sigma\left(g|x|^{-1 / 2}(|p|+1)^{-1}|x|^{-1 / 2} g\right)=[0, \pi / 2] .
\end{gathered}
$$

Together with (11) and (12) this implies $\left(\mu=\max _{k} \mu_{k}\right)$

$$
\sigma_{\text {ess }}\left(V_{\mathbf{R}}^{1 / 2}(|p|+1)^{-1} V_{\mathbf{R}}^{1 / 2}\right)=[0, \mu] \text {. }
$$

With the help of this proposition we can now prove Theorem 1 .

Proof of Theorem 1 . We have $(s-\lim =$ strong limit $)$

$$
\begin{aligned}
& s-\lim _{\lambda \rightarrow 0} V_{\lambda \mathbf{R}}^{1 / 2}\left[\left(p^{2}+m^{2}\right)^{1 / 2}+|E|\right]^{-1} V_{\lambda \mathbf{R}}^{1 / 2} \\
& \quad=(2 M / \pi)|x|^{-1 / 2}\left[\left(p^{2}+m^{2}\right)^{1 / 2}+|E|\right]^{-1}|x|^{-1 / 2}
\end{aligned}
$$

[all these operators are bounded, and $V_{\lambda \mathbf{R}} \underset{\lambda \rightarrow 0}{\longrightarrow}(2 M / \pi)|x|^{-1}$ a.e., where $\left.M=\sum_{k} \mu_{k}\right]$.

For $\lambda \rightarrow 0$, we have

$$
\sigma_{\mathrm{ess}}\left(V_{\lambda \mathbf{R}}^{1 / 2}\left[\left(p^{2}+m^{2}\right)^{1 / 2}+|E|\right]^{-1} V_{\lambda \mathbf{R}}^{1 / 2}\right)=[0, \mu],
$$

while

$$
\sigma_{\text {ess }}\left((2 M / \pi)|x|^{-1 / 2}\left[\left(p^{2}+m^{2}\right)^{1 / 2}+|E|\right]^{-1}|x|^{-1 / 2}\right)=[0, M] .
$$

Since $\mu \leqq 1<M$, the strong convergence (14) implies that every $e \in(1, M]$ can be written as $e=\lim _{n \rightarrow \infty} e_{n}$, with $e_{n}$ an eigenvalue larger than 1 of

$$
V_{\mathbf{R} / n}^{1 / 2}\left[\left(p^{2}+m^{2}\right)^{1 / 2}+|E|\right]^{-1} V_{\mathbf{R} / n}^{1 / 2}
$$


(see Theorem VIII.24 in [5]). One easily sees that this implies

$$
\lim _{n \rightarrow \infty} \#\left\{e>1 ; e \text { eigenvalue of } V_{\mathbf{R} / n}^{1 / 2}\left[\left(p^{2}+m^{2}\right)^{1 / 2}+|E|\right]^{-1} V_{\mathbf{R} / n}^{1 / 2}\right\}=\infty \text {. }
$$

By the Birman-Schwinger principle this implies $(E<0)$

$$
\lim _{n \rightarrow \infty} \#\left\{e<E ; e \text { eigenvalue of }\left(p^{2}+m^{2}\right)^{1 / 2}-V_{\mathbf{R} / n}\right\}=\infty \text {. }
$$

Since $\left(p^{2}+m^{2}\right)^{1 / 2}-V_{\lambda \mathbf{R}}$ is unitarily equivalent with

$$
\lambda^{-1}\left[\left(p^{2}+\lambda^{2} m^{2}\right)^{1 / 2}-V_{\mathbf{R}}\right]
$$

the negative eigenvalues of $\left(p^{2}+m^{2}\right)^{1 / 2}-V_{\lambda \mathbf{R}}$ decrease monotonically as $\lambda \backslash 0$. Together with (15) this proves the theorem.

Remarks. 1. Using the continuity in $\lambda$ of the eigenvalues of $\left(p^{2}+m^{2}\right)^{1 / 2}-V_{\lambda \mathbf{R}}$ (which follows from the unitary equivalence of this operator with $\left.\lambda^{-1}\left[\left(p^{2}+\lambda^{2} m^{2}\right)^{1 / 2}-V_{\mathbf{R}}\right]\right)$, one can rewrite the conclusion of Theorem 1 as:

$$
\forall E<0, \quad \forall \lambda_{0}: \#\left\{\lambda<\lambda_{0} ; E \in \sigma\left(\left(p^{2}+m^{2}\right)^{1 / 2}-V_{\lambda \mathbf{R}}\right)\right\}=\infty .
$$

It is in this form that the analogous theorem for Dirac operators was stated in [4].

2. In order for the conclusion of Theorem 1 to hold, it is not really necessary that all the internuclear distances shrink to zero, nor that the shrinking is an orderly, simultaneous contraction. The argument of the proof also works in the following situation. Let $S$ be any subset of $\{1, \ldots, K\}$ such that $\sum_{k \in S} \mu_{k}>1$. Define

$$
d_{S}=\max \left\{\left|R_{k}-R_{l}\right| ; k, l \in S\right\} .
$$

Then, for any $E<0$,

$$
\#\left\{e<E ; e \text { eigenvalue of }\left(p^{2}+m^{2}\right)^{1 / 2}-V_{\mathbf{R}}\right\}
$$

tends to $\infty$ as $d_{S}$ tends to zero.

3. If we put $m=0$, we find that $|p|-V_{\lambda \mathbf{R}}$ and $\lambda^{-1}\left(|p|-V_{\mathbf{R}}\right)$ are unitarily equivalent. The number of negative eigenvalues of $|p|-V_{\lambda \mathbf{R}}$ is therefore independent of $\lambda$, which implies that $|p|-V_{\mathbf{R}}$ has infinitely many negative eigenvalues if $\sum_{k=1}^{K} \mu_{k}>1$.

\section{A Lower Bound on $\boldsymbol{H}_{K}$}

Following the same strategy as in [3], we shall first prove (7) in the case where all the $Z_{k}$ are equal, and then use a concavity argument to extend this result to arbitrary $Z_{k}$.

Proposition. Take $6 \leqq Z \leqq Z_{\text {crit }}=(2 / \pi) \alpha^{-1}$. Then

$$
\left(p^{2}+m^{2}\right)^{1 / 2}-Z \alpha \sum_{k=1}^{K}\left|x-R_{k}\right|^{-1}+Z^{2} \alpha \sum_{\substack{k, l=1 \\ k<l}}^{K}\left|R_{k}-R_{l}\right|^{-1} \geqq m\left[1-Z^{2} / Z_{\mathrm{crit}}^{2}\right]^{1 / 2}
$$


Proof. The following inequality [stronger than (4)] was proved in [3]:

$$
|p|-(2 / \pi)\left[\sum_{k=1}^{K}\left|x-R_{k}\right|^{-1}-6 \sum_{\substack{k, l=1 \\ k<l}}^{K}\left|R_{k}-R_{l}\right|^{-1}\right] \geqq 0 .
$$

This implies

$$
|p|^{-1 / 2}\left(Z \alpha \sum_{k=1}^{K}\left|x-R_{k}\right|^{-1}-6 Z \alpha \sum_{\substack{k, l=1 \\ k<l}}^{K}\left|R_{k}-R_{l}\right|^{-1}\right)|p|^{-1 / 2} \leqq Z / Z_{\text {crit }} .
$$

With the notation

$$
v(x)=Z \alpha \sum_{k=1}^{K}\left|x-R_{k}\right|^{-1}-Z^{2} \alpha \sum_{\substack{k, l=1 \\ k<l}}^{K}\left|R_{k}-R_{l}\right|^{-1},
$$

we have therefore

$$
Z \geqq 6 \Rightarrow|p|^{-1 / 2} v|p|^{-1 / 2} \leqq Z / Z_{\text {crit }} .
$$

For $Z=Z_{\text {crit }}$, (16) was already proved in [3]. We shall therefore assume $Z<Z_{\text {crit }}$.

We now borrow an argument from [1] to prove (16). Fix $\lambda \in[0, m)$, and define $H_{0}=\left(p^{2}+m^{2}\right)^{1 / 2}$. Then

$$
\begin{aligned}
B & \equiv\left(H_{0}-\lambda\right)^{-1 / 2} v\left(H_{0}-\lambda\right)^{-1 / 2} \leqq\left(Z / Z_{\text {crit }}\right)\left\|\left(H_{0}-\lambda\right)^{-1 / 2}|p|^{1 / 2}\right\|^{2} \\
& =\left(Z / Z_{\text {crit }}\right) m\left(m^{2}-\lambda^{2}\right)^{-1 / 2} .
\end{aligned}
$$

This implies that $\mathbb{1}-B$ is invertible for $\lambda<m\left[1-Z^{2} / Z_{\text {crit }}^{2}\right]^{1 / 2}$, hence that

$$
\left(H_{0}-v-\lambda\right)^{-1}=\left(H_{0}-\lambda\right)^{-1 / 2}(\mathbb{1}-B)^{-1}\left(H_{0}-\lambda\right)^{-1 / 2}
$$

is a bounded operator. We have therefore proved that

$$
\left[0, m\left(1-Z^{2} / Z_{\text {crit }}^{2}\right)^{1 / 2}\right) \subset \varrho\left(H_{0}-v\right) .
$$

Since $H_{0}-v \geqq 0$ by (4), this proves (16).

By means of a concavity argument we can extend this to obtain the following result:

Proposition. Fix $\tilde{Z}$, with $6 \leqq \tilde{Z} \leqq Z_{\text {crit }}$. Choose $Z_{1}, \ldots, Z_{K}$ such that $0 \leqq Z_{k} \leqq \tilde{Z}$ for all k. Then

$$
\left(p^{2}+m^{2}\right)^{1 / 2}-\sum_{k=1}^{K} Z_{k} \alpha\left|x-R_{k}\right|^{-1}+\sum_{k<l} Z_{k} Z_{l} \alpha\left|R_{k}-R_{l}\right|^{-1} \geqq m\left(1-\tilde{Z}^{2} / Z_{\mathrm{crit}}^{2}\right)^{1 / 2} .
$$

Proof. We use the notation $\mathbf{Z}$ for the set $\left\{Z_{1}, \ldots, Z_{K}\right\}$. Define for all $\mathbf{Z} \in[0, \tilde{Z}]^{K}$,

$$
G(\mathbf{Z})=\inf \operatorname{spec}\left\{\left(p^{2}+m^{2}\right)^{1 / 2}-\sum_{k=1}^{K} Z_{k} \alpha\left|x-R_{k}\right|^{-1}-m\left(1-\tilde{Z}^{2} / Z_{\text {crit }}^{2}\right)^{1 / 2}\right\} .
$$

$G(\mathbf{Z})$ is a jointly concave function in the $Z_{k}$. According to (16) we have at every cornerpoint $\mathbf{P}$ of the cube $[0, \tilde{Z}]^{K}$,

$$
G(\mathbf{P}) \geqq-\sum_{k<l} P_{k} P_{l} \alpha\left|R_{k}-R_{l}\right|^{-1}
$$


By concavity it follows that (see Lemma 2.3 in [3])

$$
G(\mathbf{Z}) \geqq-\sum_{k<l} Z_{k} Z_{l} \alpha\left|R_{k}-R_{l}\right|^{-1} \quad \text { for all } \quad \mathbf{Z} \in[0, \tilde{Z}]^{K}
$$

Theorem 2, as it was stated in the introduction, is essentially a reformulation of this last proposition: for given $Z_{1}, \ldots, Z_{K}$, with $Z_{\max }=\max _{k} Z_{k} \geqq 6$, we choose $\tilde{Z}=Z_{\max } ;$ the theorem then follows immediately.

If $Z_{\max }<6$, one can always choose $\tilde{Z}=6$, which gives for $Z_{1}, \ldots, Z_{K}<6$ :

$$
\left(p^{2}+m^{2}\right)^{1 / 2}-\sum_{k=1}^{K} Z_{k} \alpha\left|x-R_{k}\right|^{-1}+\sum_{k<l} Z_{k} Z_{l} \alpha\left|R_{k}-R_{l}\right|^{-1} \geqq m\left(1-36 / Z_{\mathrm{crit}}^{2}\right)^{1 / 2} .(18
$$

If all the $Z_{k}$ are chosen equal, $Z_{k}=Z$, and the limit $Z \rightarrow 0$ is taken, one expects that the ground state $E_{0}\left(H_{K}\right)$ approaches $m: \lim _{Z \rightarrow 0} E_{0}\left(H_{K}\right)=m$, physically. This is not reflected by the lower bound (18). Actually, we can do slightly better than (18). If we define $G(\mathbf{Z})$ as above, we obviously have

$$
G(\mathbf{O}) \geqq m(1-a), \quad G\left(\mathbf{Q}_{j}\right) \geqq 0, \quad \text { where } \quad a=\left(1-36 / Z_{\text {crit }}^{2}\right)^{1 / 2}
$$

(we assume $Z_{k} \leqq 6$, for all $k$ ), and where $\mathbf{O}, \mathbf{Q}_{j}$ are $K$-tuples defined as $\mathbf{O}=(0, \ldots, 0)$ (all entries zero), $\mathbf{Q}_{j}=(0, \ldots, 0,6,0, \ldots, 0)$ (all entries zero except the $j$ th which equals 6). By concavity this implies $\left(Z_{k} \leqq 6\right)$

$$
G(\mathbf{Z}) \geqq m(1-a)\left(1-\sum_{k} Z_{k} / 6\right) \text { if } \sum_{k} Z_{k} \leqq 6,
$$

hence

$$
G(\mathbf{Z}) \geqq-\sum_{k<l} Z_{k} Z_{l} \alpha\left|R_{k}-R_{l}\right|^{-1}+m(1-a) \max \left(1-\sum_{k} Z_{k} / 6,0\right) .
$$

For $Z_{1}, \ldots, Z_{K}<6$, this can be rewritten as:

$$
\begin{aligned}
& \left(p^{2}+m^{2}\right)^{1 / 2}-\sum_{k=1}^{K} Z_{k} \alpha\left|x-R_{k}\right|^{-1}+\sum_{k<l} Z_{k} Z_{l} \alpha\left|R_{k}-R_{l}\right|^{-1} \\
& \geqq m\left\{1-\min \left(1, \sum_{k} Z_{k} / 6\right)\left[1-\left(1-36 / Z_{\mathrm{crit}}^{2}\right)^{1 / 2}\right]\right\} .
\end{aligned}
$$

This lower bound has the advantage of tending to $m$ when all the $Z_{k}$ are equal and tend to zero. However, we strongly suspect that (19) is not optimal; physically one would believe that Theorem 2 holds without restriction on $\max Z_{k}$.

Remark. Note that (17) implies the existence of a $c$-independent lower bound for $H_{K}(c)-m c^{2}$. Re-introducing $c$, we see from (17) that

$$
\begin{aligned}
& \left(p^{2} c^{2}+m^{2} c^{4}\right)^{1 / 2}-m c^{2}-\sum_{k=1}^{K} Z_{k} e^{2}\left|x-R_{k}\right|^{-1} \\
& \quad \geqq-\sum_{k<l} Z_{k} Z_{l} e^{2}\left|R_{k}-R_{l}\right|^{-1}+m c^{2}\left[\left(1-a^{2} c^{-2}\right)^{1 / 2}-1\right],
\end{aligned}
$$

with $a=c \max \left(6,\left\{Z_{k}\right\}\right) / Z_{\text {crit }}=\left(e^{2} \pi / 2 \hbar\right) \max \left(6,\left\{Z_{k}\right\}\right)$. Expression (20) implies

$$
H_{K}(c)-m c^{2} \geqq-\sum_{k<l} Z_{k} Z_{l} e^{2}\left|R_{k}-R_{l}\right|^{-1}-m a^{2} \equiv-b(\mathbf{Z}, \mathbf{R}) .
$$




\section{The Nonrelativistic Limit}

The naive way of obtaining the nonrelativistic limit of $H_{K}(c)-m c^{2}$ would be to write

$$
\left(p^{2} c^{2}+m^{2} c^{4}\right)^{1 / 2}-m c^{2}=p^{2} / 2 m+O\left(c^{-2}\right)
$$

and to apply perturbation theory. This is actually what the Foldy-Wouthuysen approach [5] does for the more complicated Dirac case. One readily sees however that the next term in the development (22) is - $-\left(p^{4} / 8 m^{3}\right) c^{-2}$, which is far too singular to be considered as a "perturbation" of the zeroth order term $p^{2} / 2 m$. The same problem occurs in the Foldy-Wouthuysen derivation of the nonrelativistic limit of the Dirac Hamiltonian. Recently Gesztesy, Grosse, and Thaller showed that the proper way of looking at the nonrelativistic limit of a Dirac Hamiltonian is to study the resolvent. They showed that the Dirac resolvent is holomorphic in $c^{-1}$ around its nonrelativistic limit $c^{-1}=0$ [7], and obtained powerful results concerning the analyticity of the eigenvalues in $c^{-2}$, and an explicit formula, for the first order relativistic correction term to the nonrelativistic eigenvalues [8] (simpler than Foldy-Wouthuysen!). The same clue (i.e. studying the resolvent instead of the Hamiltonian itself) works in our present case. We have

Proposition. Let $H_{K}(c), H_{K}^{0}$ be defined as in (1), (8) respectively. Then for all $\mathbf{Z}, \mathbf{R}$, one can find $z \in \mathbb{C}, c_{0}$, and $k \geqq 0$ such that $c \geqq c_{0} \Rightarrow z$ is in the resolvent sets of $H_{K}(c)-m c^{2}$ and $H_{K}^{0}$, and

$$
\left\|\left(H_{K}^{0}-z\right)^{-1}-\left(H_{K}(c)-m c^{2}-z\right)^{-1}\right\| \leqq k c^{-1} .
$$

Proof. Define $T^{0}=p^{2} / 2 m, T(c)=\left(p^{2} c^{2}+m^{2} c^{4}\right)^{1 / 2}-m c^{2}, V_{\mathbf{Z}}(x)=\sum_{k} Z_{k} e^{2}\left|x-R_{k}\right|^{-1}$. Obviously $V_{\lambda \mathbf{Z}}=\hat{\lambda} V_{\mathbf{z}}$. Fix $\mathbf{Z}, \mathbf{R}$. Choose $\lambda>1$, and define $c_{0}=\left(\pi e^{2} / 2 \hbar\right) \lambda \max _{k} Z_{k}$. We have, using (21),

$$
c \geqq c_{0} \Rightarrow T(c)-\lambda V_{\mathbf{Z}}=T(c)-V_{\lambda \mathbf{Z}} \geqq-b(\lambda \mathbf{Z}, \mathbf{R}) .
$$

[The value of $c_{0}$ was chosen so as to ensure that $V_{\lambda \mathbf{Z}}$ is still $T(c)$-formbounded.] Put $b=\lambda^{-1} b(\lambda \mathbf{Z}, \mathbf{R})$. Then

$$
(T(c)+\mathbb{1})^{-1 / 2}\left(V_{\mathbf{Z}}-b\right)(T(c)+\mathbb{1})^{-1 / 2} \leqq \lambda^{-1} .
$$

This implies that

$$
\mathbb{1}-(T(c)+\mathbb{1})^{-1 / 2}(V-b)(T(c)+\mathbb{1})^{-1 / 2} \text { is invertible }\left(V=V_{\mathbf{Z}}\right),
$$

with $\left\|\left[\mathbb{1}-(T(c)+\mathbb{1})^{-1 / 2}(V-b)(T(c)+\mathbb{1})^{-1 / 2}\right]^{-1}\right\| \leqq \lambda /(\lambda-1)$.

Choose $\beta \in \mathbb{R}$ large enough so that

$$
\left\|(V-b)\left(T^{0}+\mathbb{1}+i \beta\right)^{-1}\right\| \leqq \lambda^{-1},
$$

hence

$$
\left\|\left[\mathbb{1}-(V-b)\left(T^{0}+\mathbb{1}+i \beta\right)^{-1}\right]^{-1}\right\| \leqq \lambda /(\lambda-1) .
$$


Put now $z=-(b+1+i \beta)$. Then

$$
\begin{aligned}
{\left[H_{K}(c)-m c^{2}-z\right]^{-1}-\left[H_{K}^{0}-z\right]^{-1} } \\
=\left[H_{K}(c)-m c^{2}-z\right]^{-1}\left[T^{0}-T(c)\right]\left[H_{K}^{0}-z\right]^{-1} \\
=[T(c)-V+b+1+i \beta]^{-1}[T(c)-V+1+b][T(c)+\mathbb{1}]^{-1 / 2} \\
\\
\cdot\left[\mathbb{1}-(T(c)+\mathbb{1})^{-1 / 2}(V-b)(T(c)+\mathbb{1})^{-1 / 2}\right]^{-1} \\
\cdot(T(c)+\mathbb{1})^{-1 / 2}\left[T^{0}-T(c)\right]\left(T^{0}+\mathbb{1}\right)^{-1}\left(T^{0}+\mathbb{1}\right)\left(T^{0}+\mathbb{1}+i \beta\right)^{-1} \\
\\
\cdot\left[\mathbb{1}-(V-b)\left(T^{0}+\mathbb{1}+i \beta\right)^{-1}\right]^{-1} .
\end{aligned}
$$

Hence

$$
\begin{aligned}
& \left\|\left[H_{K}(c)-m c^{2}-z\right]^{-1}-\left[H_{K}^{0}-z\right]^{-1}\right\| \\
& \quad \leqq \lambda^{2} /(\lambda-1)^{2}\left\|(T(c)+\mathbb{1})^{-1 / 2}\left[T^{0}-T(c)\right]\left(T^{0}+\mathbb{1}\right)^{-1}\right\| .
\end{aligned}
$$

We have

$$
\begin{aligned}
\left\|(T(c)+\mathbb{1})^{-1 / 2}\left[T^{0}-T(c)\right]\left(T^{0}+\mathbb{1}\right)^{-1}\right\| \\
=\sup _{p \in \mathbb{R}^{3}}\left\{\left[\left(p^{2} c^{2}+m^{2} c^{4}\right)^{1 / 2}-m c^{2}+1\right]^{-1 / 2}\right. \\
\left.\quad \cdot\left[p^{2} / 2 m-\left(p^{2} c^{2}+m^{2} c^{4}\right)^{1 / 2}+m c^{2}\right]\left[1+p^{2} / 2 m\right]^{-1}\right\} \\
=\sup _{t \in \mathbb{R}_{+}}\left\{(1+t)^{-1 / 2}\left(t^{2} / 2 m c^{2}\right)\left(1+t+t^{2} / 2 m c^{2}\right)^{-1}\right\} \\
\leqq \\
\sup _{t \in \mathbb{R}_{+}}\left\{\left(t^{1 / 2} / 2 m c^{2}\right)\left(1+t / 2 m c^{2}\right)^{-1}\right\} \leqq\left(8 m c^{2}\right)^{-1 / 2}
\end{aligned}
$$

This proves (23), with $k=(8 m)^{-1 / 2} \lambda^{2} /(\lambda-1)^{2}$.

As a consequence of (23), the eigenvalues of $\left(H_{K}(c)-m c^{2}-z\right)^{-1}$ tend to the eigenvalues of $\left(H_{K}^{0}-z\right)^{-1}$ for $c \rightarrow \infty$, with a difference of order $c^{-1}$ (or less). Since the eigenvalues of $H$ correspond to the eigenvalues of the resolvent $(H-z)^{-1}$, this implies that the eigenvalues $E_{n}(c)-m c^{2}$ of $H_{K}(c)-m c^{2}$ tend to the eigenvalues $E_{n}^{0}$ of $H_{K}^{0}$, with again $\left|E_{n}(c)-m c^{2}-E_{n}^{0}\right| \leqq k_{n} c^{-1}$. This proves Theorem 3 , formulated in the introduction.

Remark. It is clear that our estimate (21) is weak when compared with the results for the Dirac operator in $[7,8]$. We have little doubt that stronger results also exist for our present operator $H_{K}(c)$. It seems improbable that the resolvent of $H_{K}(c)$ $-m c^{2}$ would still be holomorphic in $c^{-1}$, because of the presence of the square root, but it is possible that the eigenvalues still are holomorphic. We have nothing to say about this.

Acknowledgement. I would like to thank Elliott Lieb for suggesting problems 1) and 2) to me, and for several interesting discussions.

\section{References}

1. Herbst, I.: Spectral theory of the operator $\left(p^{2}+m^{2}\right)^{1 / 2}-Z e^{2} / r$. Commun. Math. Phys. 53, 285-294 (1977)

2. Weder, R.: Spectral analysis of pseudodifferential operators. J. Funct. Anal. 20, 319-337 (1975) 
3. Daubechies, I., Lieb, E.: One electron relativistic molecules with Coulomb interaction. Commun. Math. Phys. 90, 497-510 (1983)

4. Klaus, M.: Dirac operators with several Coulomb singularities. Helv. Phys. Acta 53, 463-482 (1980)

5. Reed, M., Simon, B.: Methods of modern mathematical physics, Vol. I. Functional analysis. New York: Academic Press 1972

6. Foldy, L., Wouthuysen, S.: On the Dirac theory of spin $1 / 2$ particles and its non-relativistic limit. Phys. Rev. 78, 29-36 (1950)

7. Gesztesy, F., Grosse, H., Thaller, B.: A rigorous approach to relativistic corrections of bound state energies for spin 1/2 particles. Ann. Inst. H. Poincaré 40, 159-174 (1984)

8. Gesztesy, F., Thaller, B., Grosse, H.: An efficient method for calculating relativistic corrections for spin 1/2 particles. Phys. Rev. Lett. 50, 625-628 (1983)

Communicated by B. Simon

Received January 19, 1984 
